\title{
Cutaneous larva migrans on the abdomen of a child with delayed gross motor development.
}

\section{${ }^{1}$ Thadchanamoorthy $V,{ }^{2}$ Dayasiri $K$}

${ }^{1}$ Faculty of Health Care Science, Eastern University, ${ }^{2}$ Base Hospital Mahaoya.

\begin{abstract}
Cutaneous larva migrans is a tropically acquired parasitic infection caused mostly by the larvae of Ancylostoma braziliense and Ancylostoma caninum. We report a child with an unusual presentation of cutaneous larva migrans infection on her abdominal wall. The reported child with delayed gross-motor development presented to the pediatric clinic with a history of irritable cry and poor sleep for one-month duration. She could only roll-over on cemented floor in her house which had poor infrastructure facilities and also had several pets which frequently entered into the house. Examination revealed linear erythematous lesion on abdominal wall compatible with clinical diagnosis of cutaneous larva migrans. The child responded well to a seven-day course of Albendazole.
\end{abstract}

\section{Keywords:}

Cutaneous larva migrans, Albendazole, Hookworms

\section{Introduction}

Cutaneous larva migrans (CLM) is a cutaneous dermatitis. It was first reported by the English physician, Dr. Lee in 1893 (1). This parasitic infection is caused by hook worms of dogs and cats, such as Ancylostoma braziliense and Ancylostoma caninum which are commonly found in tropical and subtropical countries $(1,2)$. Patients usually acquire the disease when they walk with bare foot, playing in the soil, or have prolonged sunbathing in contaminated soil. The lesions are usually confined to feet, buttocks and hands. It is mostly diagnosed by its typical clinical presentation - pruritic linear serpiginous lesions.

A previous study from Northern Province of Sri Lanka reported a high prevalence of CLM amongst devotees who regularly perform side-rolling rituals (3). CLM infestation involving areas other than feet, buttocks and hands is unusual in children. The authors of this report describe a child with grossmotor delay who presented with CLM infestation on the abdominal wall.

\section{Case history}

A 2-year-old child was referred to pediatric clinic following episodes of irritable cry and poor sleep for one-month duration. She had global developmental delay and epilepsy following neonatal meningitis and was under multidisciplinary team follow-up. With regard to her gross-motor development, she could only roll-over and was unable to stand or walk. Physical examination revealed a $6 \mathrm{~cm}$ long, linear serpiginous lesion in the peri-umbilical region suggestive of cutaneous larva migrans. (Figure 1) Her gross-motor developmental age was compatible with 6 to 7 months. The child had not received any anti-worm treatment in the past. Clinical history was re-visited following detection of CLM infestation at the unusual site. Child had been rolling-over on the cemented floor most of the time with minimal clothing. The family was from a poor socio-economic background and had poor housing and infrastructure facilities. They had several pets including cats and dogs which had not received any deworming treatment.

The child was treated with Albendazole and antihistamines for one-week duration. She recovered within the treatment period with significant symptomatic relief. Complete clearance of skin lesions was seen at one-month review.

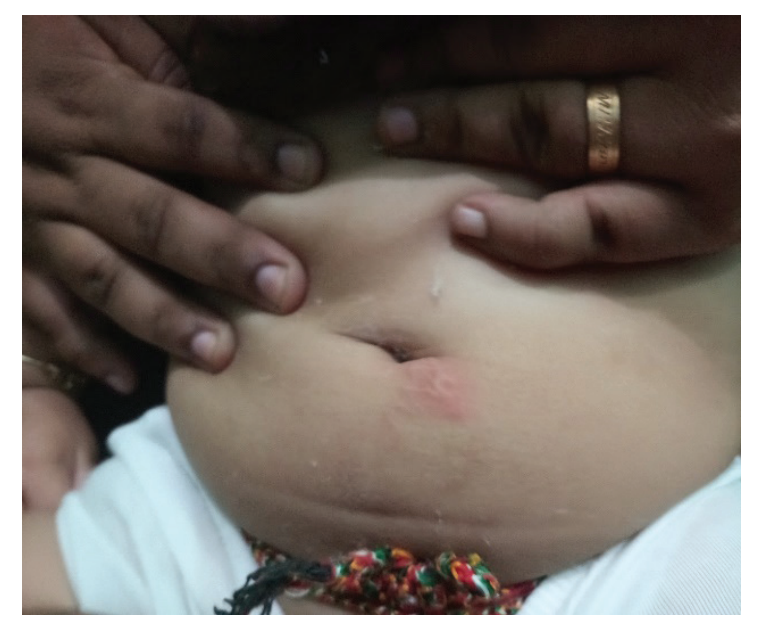

Figure-1 - Linear serpiginous lesion in the periumbilical region 


\section{Discussion}

Cutaneous larva migrans, also known as creeping eruption or sandworm disease is common in tropical and subtropical countries. It is caused by larvae of animal hookworms and rarely from other insects $(2$, $4)$. The disease is endemic in developing countries especially in resource-poor communities and also has been reported as minor outbreaks amongst tourists who returned from tropical countries (5). Children acquire the disease by walking on bare foot and by sitting and playing in the contaminated soil (2). The reported child did not have a history of lying on contaminated soil. Therefore, the most likely mechanism of acquisition of disease was frequent rolling-over on contaminated cemented floor where larva could potentially be found due to frequent movement of cats and dogs. Presence of cutaneous larva migrans on the abdomen is an infrequently reported presentation in children $(6$, 7).

The commonly affected areas in CLM infections are dorsum and sole of the feet, buttocks, pelvic waist, legs and shoulders. The characteristic clinical features are linear and/or serpiginous lesions which are pruritic. The larvae move at a rate from $2-3 \mathrm{~mm}$ to $2-3 \mathrm{~cm}$ per day. The symptoms are aggravated by inflammatory reactions and persistent symptoms disturb sleep and daily activities and make children irritable due to itchy lesions. Systemic features related to peripheral eosinophilia (Loeffler syndrome), migratory pulmonary infiltrates, and increased immunoglobulin E levels are occasionally present however they are rare (8).

The diagnosis of cutaneous larva migrans is made by clinical evaluation as it has typical lesions and generally no investigations are necessary. Although the lesions are self-limited, secondary bacterial infection and severe pruritus may demand pharmacological treatment. A single dose of ivermectin ( $200 \mu \mathrm{g}$ per $\mathrm{kg}$ bodyweight) is usually enough to destroy the migrating larvae and relieve itching. Oral albendazole (200-400 mg daily) is similarly very effective in eradicating larva and adult worms. It should be given for 5-7 days, although longer regimens are needed in severe chronic infections. Albendazole is well-tolerated by children (5). The prognosis is good with early and accurate diagnosis and treatment. The lesions resolve over 4 to 8 weeks as humans are accidental, dead-end hosts. Rarely, it might take up to one year for complete resolution in severe cases (9). As the index child had uncomplicated CLM, the lesions completely responded to seven-day-course of Albendazole with no recurrence.

It is important to deworm all pets within house hold to prevent CLM. Children should be taught to follow general hygienic practices and parents should keep the environment clean especially floor areas within household (1). Mother of this child was also advised to deworm pets at house in consultation with veterinary surgeon.

\section{Conclusion}

Cutaneous larva migrans is a clinical diagnosis due to its typical appearance and needs prompt treatment to prevent complications and to reduce troublesome symptoms including poor sleep and irritability. Children with gross-motor developmental issues and those who frequently play barefoot on contaminated floors may be at high risk for CLM. Pets should be periodically dewormed with mebendazole to prevent CLM.

\section{Ethical Considerations}

Written informed consent was obtained from the patient for publication and accompanying image

\section{References}

1. Neafie RC, Meyers WM. Cutaneous larva migrans. In: Strickland GT ed. Hunters Tropical Medicine and Emerging Infectious Diseases, 8th edn. Philadelphia: Sounders 2000: 797-799

2. Jelinek T, Maiwald H, Nothdurft HD, Löscher T, 1994. Cutaneous larva migrans in travelers: synopsis of histories, symptoms, and treatment of 98 patients. Clin Infect Dis 19: 1062-1066.https:// doi.org/10.1093/clinids/19.6.1062

3. Kannathasan S, Murugananthan A, Rajeshkannan N, de Silva NR (2012) Cutaneous Larva Migrans among Devotees of the Nallur Temple in Jaffna, Sri Lanka. PLoS ONE 7(1): e30516. https://doi. org/10.1371/journal.pone.0030516

4. Prayaga S, Mannepuli GB. A tropical souvenir not worth picking up. Cleve Clin J Med. 2006;73:458459.https://doi.org/10.3949/ccjm.73.5.458

5. Heukelbach J, Feldmeier H. Epidemiological and clinical characteristics of hookworm- 
related cutaneous larva migrans. Lancet Infect Dis. 2008;8:302-309. doi: 10.1016/S14733099(08)70098-7.https://doi.org/10.1016/S14733099(08)70098-7

6. Prashanth SN, Kumar KJ, Kumar MGA. Cutaneous larva migrans in a child. Sudan J Paediatr. 2017;17(1):66-67.

7. Dong-Lai Ma, Sergio Vano-Galvan Creeping Eruption --- Cutaneous Larva Migrans. N Engl J Med. 2016;374: e16.
8. Veraldi S, Rizzitelli G. Effectiveness of a new therapeutic regimen with albendazole in cutaneous larva migrans. Eur J Dermatol. 1999;9:352-353.

9. Cutaneous larva migrans

10. Kaliaperumal Karthikeyan, Devinder Mohan Thappa

11. Department of Dermatology and STD, Jawaharlal Institute of Postgraduate Medical Education and Research (JIPMER), Pondicherry - 605 006, India year2002/volume:68/issue:5?page252-258. 